Artigo Original

\title{
Efeito da familiarização na estabilização dos valores de 1RM para homens e mulheres
}

\author{
Carla Silva-Batista \\ Valmor Tricoli \\ Gilberto Candido Laurentino \\ Mauro Alexandre Benites Batista \\ Nilo Massaru Okuno \\ Carlos Ugrinowitsch
}

\section{Laboratório de Adaptações Neuromusculares ao Treinamento de força, Escola de Educação Física e Esporte, Universidade de São Paulo, SP, Brasil}

\begin{abstract}
Resumo: O objetivo deste estudo foi verificar o número de sessões de familiarização necessárias para estabilização da carga do teste de uma repetição máxima (1RM) no exercício agachamento em homens e mulheres. Oito homens ( $25 \pm 4$ anos) e oito mulheres (20 \pm 1anos) foram submetidos a cinco sessões experimentais. ANOVA e plotagem de Bland-Altman foram utilizadas para comparar a carga de 1RM entre as sessões $(p<0,05)$. A estabilização da carga ocorreu na quarta sessão para os homens e na terceira para as mulheres, com aumentos significantes da força absoluta e relativa da primeira à quarta (17kg e $19 \%)$ e da primeira à terceira (9kg e 14\%) sessões, respectivamente. Porém, não houve diferença significante na força relativa entre os gêneros. De acordo com nossos resultados, indivíduos inexperientes em treinamento de força atingem alta reprodutibilidade ao teste de 1RM no exercício agachamento, após realizar três a quatro sessões de familiarização.
\end{abstract}

Palavras-chave: Reprodutibilidade. Treinamento de força. Avaliação da força muscular.

\section{Effect of familiarization on the stabilization of $1 R M$ values for men and women}

\begin{abstract}
The purpose of this study was to determine the number of familiarization sessions for stabilizing the load of one repetition maximum (1RM) tests on the squat exercise in men and women. Eight men (25 \pm 4 years) and eight women (20 \pm 1 years) underwent five experimental sessions. ANOVA and Bland-Altman plotting were used to compare the 1RM load between the sessions $(p<0.05)$. The stabilization of load occurred at the fourth session for men and at the third session for women, with significantly increased in relative and absolute strength from the first to the fourth $(17 \mathrm{~kg}$ and $19 \%)$ and from the first to the third (9kg and 14\%) session, respectively. However, relative strength did not differ significantly between genders. According to our results, individuals not experienced with strength training can reach high reliability in the 1RM test in the squat exercise, after performing three or four familiarizations sessions.
\end{abstract}

Keywords: Reproducibility. Strength training. Muscle strength assessment.

\section{Introdução}

Dentre as diferentes formas de treinamento de força (TF) destaca-se a prática de exercícios isoinerciais, os quais se caracterizam pela alternância de ações musculares concêntricas e excêntricas, utilizando uma resistência fixa (RATAMESS; ALVAR; EVETOCH; HOUSH; KIBLER; KRAEMER; TRIPLETT, 2009). A prescrição do TF deve ser precedida pela mensuração do nível de força do indivíduo, não somente para o estabelecimento da carga de treinamento como também para a avaliação dos resultados obtidos após um período de treino.

Tradicionalmente, o teste de força dinâmica máxima ou teste de uma repetição máxima (1RM), tem sido utilizado para avaliar a força muscular e prescrever a intensidade da carga a ser aplicada em sessões de TF (LEVINGER; GOODMAN; HARE; JERUMS; TOIA; SELIG, 2009; NIEWIADOMSKI; LASKOWSKA; GASIOROWS; CYBULSKI; STRASZ; LANGFORT, 2008; PHILLIPS; BATTERHAM; VALENZUELA; BURKETT; RELIABILITY, 2004). Neste teste, o peso máximo mobilizado em um ciclo completo de um determinado movimento é considerado como a representação da força dinâmica máxima de um indivíduo (BROWN; WEIR, 2001; NIEWIADOMSKI; LASKOWSKA; GASIOROWS; CYBULSKI; STRASZ; LANGFORT, 2008).

Segundo Brown e Weir (2001), o aumento dos valores obtidos em um teste de 1RM pode ser 
equivocadamente interpretado como um efeito positivo do processo de treinamento, quando homens e mulheres inexperientes em TF são avaliados, devido à maior variabilidade intraindivíduos. Desse modo, a aplicação de sessões de familiarização aos testes de 1RM torna-se necessária, para minimizar 0 efeito de aprendizagem do teste, produzir avaliações confiáveis e não superestimar os ganhos de força (BROWN; WEIR, 2001; REINKING; BOCKRATHPUGLIESE; WORRELL; KEGERREIS; MILLERSAYERS; FARR, 1996; RYDWIK; KARLSSON; FRANDIN; AKNER, 2007).

Ploutz-Snyder e Giamis (2001) demonstraram a estabilização da carga para mulheres jovens inexperientes em TF na quarta sessão de familiarização de um exercício uniarticular para o membro inferior. Contudo, a estabilização da força não foi verificada mesmo após quatro (CRONIN; HENDERSON, 2004) e três sessões de testes (BENTON; SWAN; PETERSON, 2009) para homens e mulheres jovens, respectivamente, sem experiência prévia em TF, em exercício multiarticular para o membro inferior. Essa discrepância entre estes estudos deve-se ao grau de complexidade dos exercícios utilizados e à variabilidade intra-indivíduos entre os testes de 1RM, uma vez que em homens e mulheres, experientes em TF, foi verificada a estabilização da carga utilizando um exercício com um maior grau de complexidade como o agachamento, entre quatro (DIAS; CYRINO; SALVADOR; CALDEIRA; NAKAMURA; PAPST; BRUNA; GURJÃO, 2005) e três sessões (SOARESCALDEIRA; DIAS; OKUNO; CYRINO; GURJÃO; PLOUTZ-SNYDER, 2009) respectivamente.

Porém, ainda não está claro o número de sessões de familiarização necessário para estabilização da carga de membros inferiores de homens e mulheres jovens e inexperientes em TF na realização de um exercício multiarticular. Frente ao exposto, nossa hipótese é que para um exercício complexo como o agachamento, homens e mulheres inexperientes em TF necessitarão de várias sessões de familiarização para a estabilização da carga de 1RM. Assim, o objetivo deste estudo foi verificar o número de sessões de familiarização necessárias para estabilização da carga do teste de 1RM, usando o exercício agachamento, em homens e mulheres sem experiência em TF.

\section{Materiais e Métodos}

\section{Amostra}

Vinte indivíduos, dez de cada gênero, foram selecionados para participar deste estudo. No entanto, apenas oito homens (idade 25,4 \pm 4,0 anos, peso corporal $74,8 \pm 13,6 \mathrm{~kg}$, estatura 176,4 $\pm 11,3 \mathrm{~cm}$, ) e 8 mulheres $(20,4 \pm 1,7$ anos, $58,1 \pm$ $7,4 \mathrm{~kg}, 163,3 \pm 6,8 \mathrm{~cm}$ ) realizaram todas as sessões experimentais. Como critérios de inclusão, os participantes deveriam ter entre $18 \mathrm{e}$ 30 anos de idade, ser sedentários ou praticar atividade física menos que duas vezes por semana, porém não terem experiência em TF. Todos participantes foram esclarecidos sobre os objetivos, riscos e benefícios decorrentes da participação no estudo, antes de assinarem um termo de consentimento livre e esclarecido, aprovado pelo comitê de ética local.

\section{Desenho Experimental}

A força dinâmica máxima foi avaliada usando o teste de 1RM no exercício agachamento com barra guiada (aparelho Smith, Cybex). Devido à complexidade do exercício agachamento (cadeia cinética fechada) que exige a coordenação das articulações do quadril, joelhos e tornozelos e requer grande habilidade do executante, e ao fato dos participantes não possuírem experiência em TF, foi realizada uma sessão prévia com 0 objetivo de ensinar aos sujeitos a técnica do movimento. Nessa sessão, os indivíduos realizaram duas séries de 15 repetições com intervalo entre elas de dois minutos, usando como sobrecarga apenas a barra $(20 \mathrm{~kg})$ do aparelho. Através de um goniômetro manual, aferiu-se a profundidade do agachamento na qual os sujeitos atingiam $90^{\circ}$ de flexão no joelho. Essa informação foi utilizada para controlar a profundidade do exercício agachamento durante e entre sessões de avaliações subseqüentes. As instruções oferecidas e os procedimentos adotados em relação ao posicionamento no aparelho Smith, estão detalhados na seção que descreve a avaliação da força dinâmica máxima. Após essa sessão inicial, todos os sujeitos foram submetidos a cinco sessões de familiarização no exercício agachamento, com intervalo de 72 horas entre as sessões.

\section{Avaliação da Força Dinâmica Máxima}

Para a realização do teste de $1 \mathrm{RM}$ no exercício agachamento, foram seguidas as orientações da Sociedade Americana de 
Fisiologistas do Exercício (BROWN; WEIR, 2001). Os participantes realizaram um aquecimento geral em esteira rolante a $9 \mathrm{~km} / \mathrm{h}$ durante cinco minutos, seguido de dois minutos de descanso. $\mathrm{Na}$ sequência, realizaram exercício de alongamento estático de baixa intensidade para a musculatura dos membros inferiores (em pé, membros inferiores unidos, membros superiores ao longo do corpo, flexionaram o tronco sobre os membros inferiores segurando na perna ou onde alcançassem). Foram realizadas duas séries de 15 segundos de duração, com intervalo de 30 segundos entre as séries. Posteriormente, os sujeitos executaram um aquecimento específico composto de uma série de cinco repetições com $50 \%$ da carga estimada para $1 \mathrm{RM}$ no exercício agachamento, e uma segunda série, de três repetições, com aproximadamente $70 \%$ dessa carga. A carga estimada para 1RM correspondia ao peso corporal do indivíduo, e foi utilizada na primeira tentativa da primeira sessão de avaliação.

Os sujeitos tiveram um máximo de cinco tentativas para determinação da carga de 1RM, em cada sessão. A primeira tentativa ocorria três minutos após a realização do aquecimento, e intervalos de descanso de igual duração eram observados entre as tentativas. Para uma tentativa ser considerada válida, o sujeito deveria realizar um ciclo completo de movimento, iniciando e finalizando $o$ exercício de agachamento na posição ereta. $O$ ciclo de movimento teve duração de quatro segundos, dois para a fase excêntrica (fase inicial), dois para a concêntrica (fase final). No ponto intermediário do ciclo de movimento os joelhos deveriam atingir $90^{\circ}$ graus de flexão antes de iniciar a fase concêntrica (final). Os sujeitos foram orientados a encostarem-se a caixas de madeira (steps) de altura regulável que foram posicionadas atrás dos mesmos, para controlar o grau de flexão dos membros inferiores durante o exercício de agachamento. Essa altura foi determinada na primeira visita ao laboratório. Além disso, fitas adesivas foram coladas no chão para a correta reprodução do posicionamento dos pés durante $\mathrm{e}$ entre as sessões experimentais (Figura 1).

A magnitude dos incrementos de carga entre tentativas era determinada de acordo com a percepção subjetiva de esforço do indivíduo, avaliada nos intervalos entre tentativas através da escala de Borg (1998). O menor incremento de carga entre tentativas foi de $5 \mathrm{~kg}$, para ambos os gêneros, mas os maiores incrementos variaram entre $5-10 \mathrm{~kg}$ para as mulheres e, entre $5-20 \mathrm{~kg}$, para os homens. Considerava-se como 1RM a maior carga suportada realizando um ciclo completo de movimento no exercício de agachamento, com técnica apropriada.

Todas as sessões experimentais foram acompanhadas por dois avaliadores, que encorajavam verbalmente os participantes durante a realização das tentativas. Os avaliadores se esforçaram para manter o mesmo padrão de encorajamento em todos os testes.

Figura 1. Posições inicial e final do exercício agachamento no Smith.

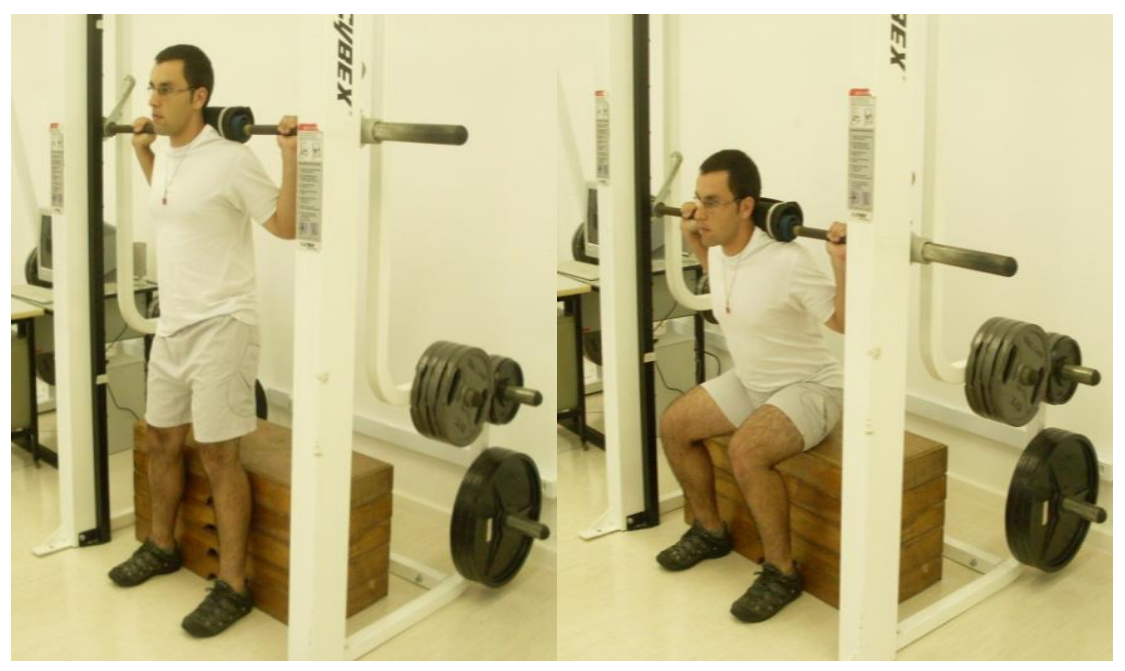

\section{Análise Estatística}

Os resultados estão apresentados com estatística descritiva (média \pm desvio padrão). 0 teste $\mathrm{t}$ de Student para amostras independentes foi utilizado para comparar os gêneros em suas variáveis descritivas. ANOVA para medidas repetidas foi utilizada para as comparações de 
$1 \mathrm{RM}$ entre as sessões de avaliação no exercício agachamento. O teste post hoc de Bonferroni foi aplicado para identificar as diferenças nas variáveis quando os valores de $\mathrm{F}$ encontrados foram significantes. Em adição, a estabilidade dos valores de 1RM obtidos nas diferentes sessões foi também analisada por meio da plotagem de Bland-Altman. O nível de significância adotado foi de $p<0,05$. As análises estatísticas foram realizadas por meio do pacote computacional SPSS 15.0 e Medcalc 9.2.0.1.

Tabela 1. Valores de $1 \mathrm{RM}(\mathrm{kg})$ no exercício agachamento para todas as sessões experimentais, para os homens e as mulheres (média $\pm \mathrm{DP}$ ).

\begin{tabular}{ccc}
\hline & HOMENS $(\mathbf{n}=\mathbf{8})$ & MULHERES $(\mathbf{n}=\mathbf{8})$ \\
\hline Sessão 1 & $98,8 \pm 22,6$ & $68,5 \pm 10,0$ \\
Sessão 2 & $107,0 \pm 18,8^{*}$ & $75,9 \pm 11,1^{*}$ \\
Sessão 3 & $112,8 \pm 19,7^{*} \dagger$ & $78,1 \pm 11,6^{*} \dagger$ \\
Sessão 4 & $116,0 \pm 20,8^{*}+£$ & $78,4 \pm 10,7^{*}$ \\
Sessão 5 & $116,6 \pm 20,5^{*}$ & $78,8 \pm 10,6^{*}$ \\
\hline
\end{tabular}

* Diferença significante em relação à primeira sessão; † Diferença significante em relação à sessão anterior; $£$ Diferença significante em relação ao valor de $1 \mathrm{RM}$ estabilizado nas mulheres ( $3^{a}$ sessão).

\section{Resultados}

Os valores de 1RM e a estabilização da carga no exercício agachamento em cada uma das cinco sessões para os homens e para as mulheres encontram-se na Tabela 1. Os resultados mostram que a estabilização da carga de 1RM ocorreu na quarta sessão para os homens $(p=0,11)$, e na terceira sessão para as mulheres $(p=1,01)$. A comparação entre os valores de 1RM estabilizados foram significantemente maiores para os homens $(p=$ $0,01)$ (Tabela 1). No entanto, para os valores em termos relativos à massa corporal não foi verificada diferença significante entre os valores de $1 \mathrm{RM}$ para os homens (1,5 $\mathrm{x}$ massa corporal) $\mathrm{e}$ as mulheres $(1,4 \times$ massa corporal) $(p=0,31)$.

Tabela 2. Variação dos valores de $1 \mathrm{RM}$ ( $\mathrm{kg}$ e \%) no exercício agachamento da primeira sessão para a sessão de estabilização (4⿳亠丷⿵冂丶 para homens, $3^{3}$ para mulheres), e da primeira sessão para a $5^{\underline{a}}$ sessão (última) (média \pm DP).

\begin{tabular}{cccc}
\hline & Sessões & Kg & $\%$ \\
\hline \multirow{2}{*}{ HOMENS $(n=8)$} & $1^{\text {a }}$ a $4^{\underline{a}}$ & $17,3 \pm 6,4^{*}$ & $19,0 \pm 11,0$ \\
& $1^{\text {a }}$ a $5^{\underline{a}}$ & $18,0 \pm 6,2^{*}$ & $20,0 \pm 2,3$ \\
MULHERES $(n=8)$ & $1^{\text {a }}$ a $3^{\underline{a}}$ & $9,6 \pm 2,3$ & $14,1 \pm 3,5$ \\
& $1^{\text {a }}$ a $5^{\text {a }}$ & $10,0 \pm 2,3$ & $15,0 \pm 2,1$ \\
\hline
\end{tabular}

*Diferença significante em relação às mulheres $(p<0,05)$.

Os aumentos médios das cargas de 1RM da primeira sessão até a sessão de estabilização, bem como da primeira até a última sessão de familiarização (quinta sessão), foram significantemente maiores para os homens, em relação às mulheres, na comparação de valores absolutos, mas não na comparação de valores relativos (Tabela 2). Apesar disso, o padrão de aumento da carga de 1RM ao longo das cinco sessões experimentais foi bastante similar entre sujeitos de ambos os gêneros (Figuras 2A e 2B).

Figura 2. Comportamento individual dos valores de $1 \mathrm{RM}$ nas cinco sessões experimentais no exercício agachamento para homens (A) e mulheres (B).

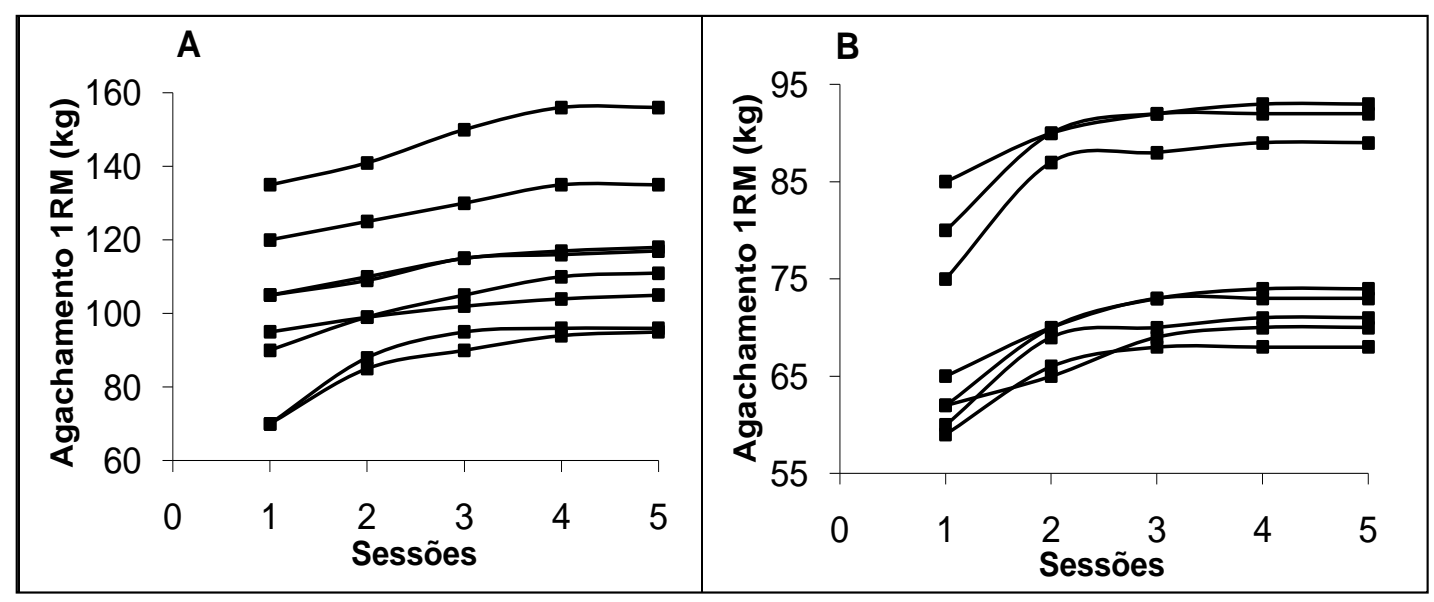


Figura 3. Plotagem de Bland-Altman mostrando as diferenças da carga $(\mathrm{kg})$ entre a primeira e segunda, a segunda e terceira, a terceira e quarta e a quarta e quinta sessão de testes de 1RM em homens.

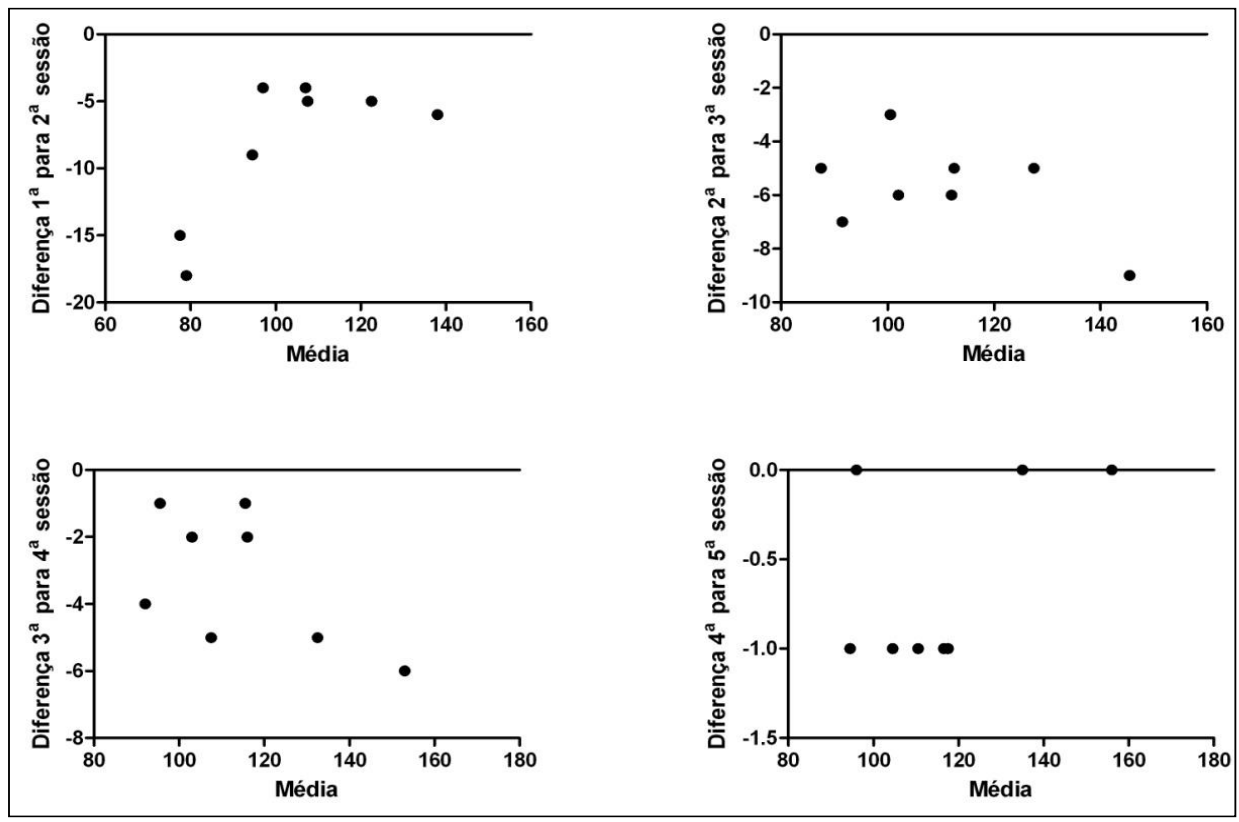

Figura 4. Plotagem de Bland-Altman mostrando as diferenças da carga $(\mathrm{kg})$ entre a primeira e segunda, a segunda e terceira, a terceira e quarta e a quarta e quinta sessão de testes de 1RM em mulheres.

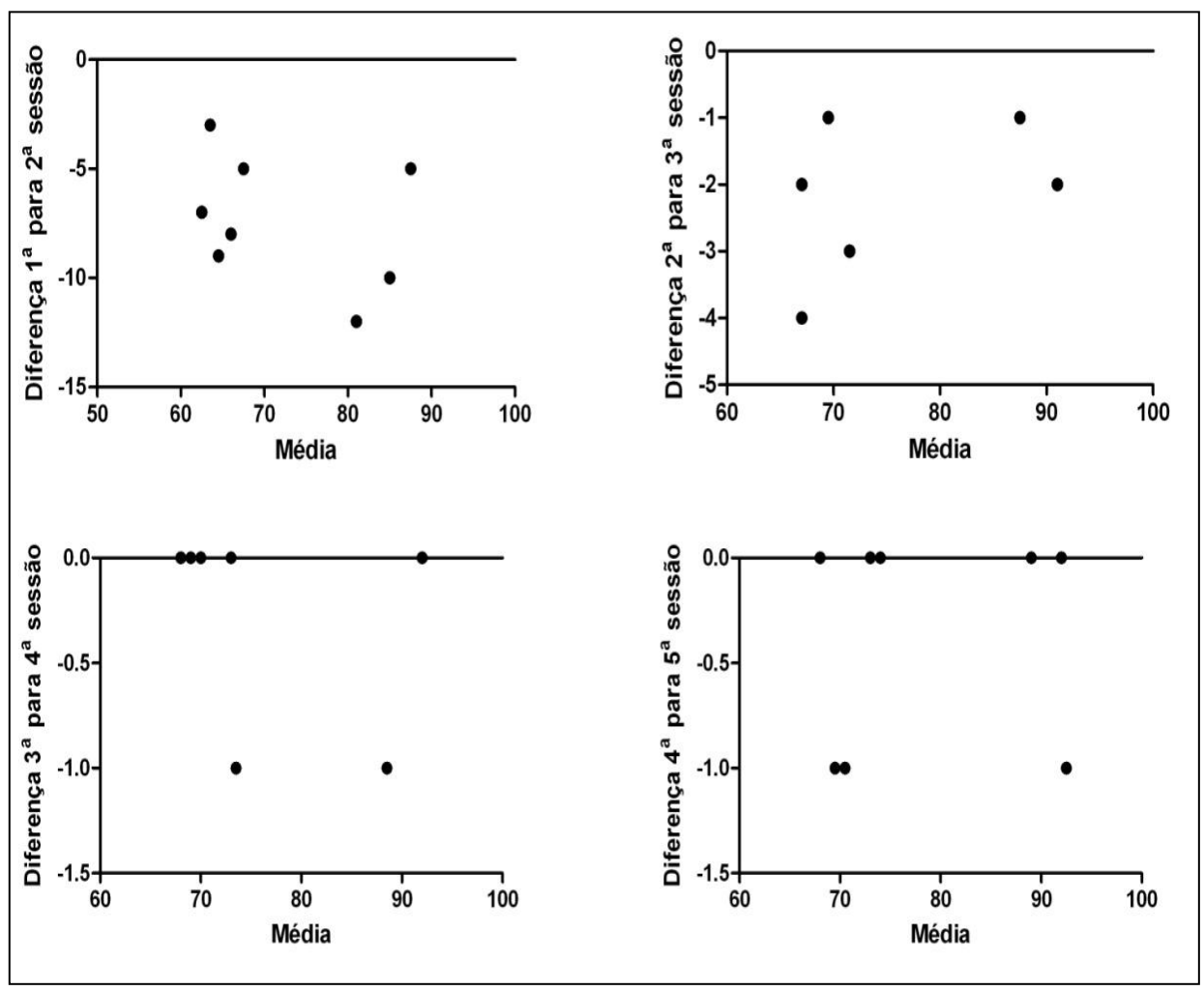

Nas Figuras 3 e 4 são ilustradas as plotagem de Bland-Altman, para os homens e para as mulheres respectivamente. Os resultados desta plotagem demonstram que a diferença do valor de $1 \mathrm{RM}$ entre as sessões sucessivas foi diminuindo progressivamente da primeira para a quinta sessão. $\mathrm{Na}$ Tabela 3 são apresentados os resultados da plotagem de Bland-Altman, onde a média das diferenças das cargas de 1RM, os limites de concordância, e o intervalo de confiança de $95 \%$ entre sessões consecutivas podem ser visualizados. Pode-se verificar que os homens atingiram uma variação menor que $1 \mathrm{~kg}$ nas cargas de dois testes consecutivos da quarta para a quinta sessão, enquanto as mulheres atingiram 
essa variação mais cedo, da terceira para a quarta sessão.

Tabela 3. Resultados da plotagem de BlandAltman entre as sessões experimentais no exercício agachamento.

\begin{tabular}{cccccc}
\hline & & \multicolumn{4}{c}{ Confronto entre as sessões } \\
\hline & & $1-2$ & $2-3$ & $3-4$ & $4-5$ \\
Média das & Homens & $-8,3$ & $-5,7$ & $-3,2$ & $-0,62$ \\
diferenças (kg) & Mulheres & $-7,4$ & $-2,2$ & $-0,2$ & $-0,3$ \\
Limites de & Homens & 21,1 & 11,5 & 7,7 & 1,9 \\
$\begin{array}{c}\text { concordância } \\
\text { (kg) }\end{array}$ & Mulheres & 14,7 & 4,5 & 1,8 & 1,9 \\
\multirow{2}{\text{IC95\%(limites}}{$\begin{array}{c}\text { Homens } \\
\text { inferior/superior) }\end{array}$} & $-18,8 / 2,3$ & $-9,2 /-2,3$ & $-7,1 / 0,6$ & $-1,6 / 0,3$ \\
& Mulheres & $-13,2 /-1,5$ & $-4,3 /-0,2$ & $-1,1 / 0,7$ & $-1,4 / 0,6$ \\
\hline
\end{tabular}

\section{Discussão}

O objetivo deste estudo foi verificar o número de sessões de familiarização necessárias para estabilização da carga usando o exercício agachamento em homens e mulheres inexperientes em TF. Os principais achados desse estudo mostram que as mulheres inexperientes em TF atingem a estabilização da carga do teste de 1RM em três sessões de familiarização, enquanto os homens precisam de quatro sessões.

A necessidade de realizar múltiplos testes antes de se obter um valor reprodutível de 1RM já havia sido verificada em outros estudos, porém através de exercícios de menor complexidade em indivíduos sem experiência prévia em TF (GURJÃO; CYRINO; CALDEIRA; NAKAMURA; OLIVEIRA; DIAS; SALVADOR, 2005; PLOUTZSNYDER; GIAMIS, 2001), ou avaliando indivíduos experientes em TF (DIAS; CYRINO; SALVADOR; CALDEIRA; NAKAMURA; PAPST; BRUNA; GURJÃO, 2005; SOARES-CALDEIRA; DIAS; OKUNO; CYRINO; GURJÃO; PLOUTZ-SNYDER, 2009). Em concordância com estes estudos, nosso experimento mostra que uma alta reprodutibilidade no teste de $1 \mathrm{RM}$ pode ser verificada ao se realizar entre três e quatro sessões de familiarização obtidas através do exercício agachamento.

Particularmente com o exercício agachamento, estudos já haviam demonstrado estabilização de 1RM, em indivíduos experientes em TF. DIAS, Cyrino, Salvador, Caldeira, Nakamura, Papst, Bruna e Gurjão (2005) observaram que quatro sessões de testes de 1RM foram suficientes para homens (24,5 $\pm 3,8$ anos) atingirem o platô da carga com aumento da força de $3,4 \%$. De maneira semelhante, Soares-Caldeira, Dias,
Okuno, Cyrino, Gurjão e Ploutz-Snyder (2009) verificaram que mulheres (21,6 $\pm 2,5$ anos) estabilizaram a carga na terceira sessão com aumento de $5,4 \%$ da força. Contudo, não se conhecia o número necessário de sessões para se estabilizar o $1 \mathrm{RM}$ em indivíduos inexperientes em TF realizando um exercício de maior complexidade (BENTON; SWAN; PETERSON, 2009; CRONIN; HENDERSON, 2004).

Contrariando nossa hipótese, nosso estudo mostra que indivíduos inexperientes em TF estabilizaram a carga do teste de 1RM com o mesmo número de sessões de indivíduos experientes, avaliados nos estudos de DIAS, Cyrino, Salvador, Caldeira, Nakamura, Papst, Bruna e Gurjão (2005) e Soares-Caldeira, Dias, Okuno, Cyrino, Gurjão e Ploutz-Snyder (2009), porém com maiores aumentos da força (Tabela 2). Isto demonstra que os ganhos de força decorrentes da aprendizagem do exercício são mais importantes para indivíduos destreinados do que treinados. Além disso, é importante considerar, que a similaridade na estabilização de 1 RM em nosso estudo, assim como nos estudos de DIAS, Cyrino, Salvador, Caldeira, Nakamura, Papst, Bruna e Gurjão (2005) e Soares-Caldeira, Dias, Okuno, Cyrino, Gurjão e Ploutz-Snyder (2009), pode ter sido influenciada pelo fato de que o exercício agachamento em nosso estudo e nos estudos citados, foi realizado no aparelho Smith, o que facilita a execução do movimento. Talvez os resultados fossem diferentes se comparados indivíduos com diferentes experiências em TF, mas executando o agachamento com barra livre.

Por outro lado, surpreendentemente, as mulheres atingiram a estabilização de 1RM no agachamento realizando uma sessão a menos de familiarização que os homens. É difícil encontrar uma explicação fisiológica para essa diferença entre gêneros, já que, relativamente à massa corporal, homens e mulheres têm o mesmo nível de força muscular (GLASS; STANTON, 2004). Uma hipótese é que as mulheres atingiram a estabilização de $1 \mathrm{RM}$ antes dos homens devido ao fato de terem menor ímpeto para a produção de força. De acordo com isso, à medida que a carga foi crescendo de sessão para sessão as mulheres teriam diminuído sua disposição em superar cargas maiores, ou seja, deixaram de se empenhar em realizar esforço máximo durante as sessões subseqüentes. Para contestar essa hipótese, poderia se usar o argumento de não ter sido verificada diferença significante entre 
gêneros, quando comparado o crescimento da carga relativa (19\% para homens, $14 \%$ para mulheres, entre as sessões em que ocorreu a estabilização, Tabela 2). Ainda, não podemos descartar a possibilidade de ocorrência de um erro tipo II nessa comparação, devido ao pequeno tamanho da amostra utilizada em nosso estudo.

Em conformidade com outros estudos, nossos dados sugerem que a avaliação da força dinâmica pode ser subestimada, caso o teste de 1RM não seja aplicado até que se verifique a estabilização da carga. Visto de outra maneira, a avaliação de 1 RM sem verificação da sua estabilização pode superestimar os ganhos de força obtidos em programas de treinamento destinados a avaliar 0 aumento da força muscular. Esta pode ser uma explicação para a ampla variação nos ganhos em força muscular reportados na literatura (27 $226 \%$ ), obtidos através de programas de TF com duração entre seis e doze semanas (CAROLAN; CAFARELLI, 1992; FIATARONE; MARKS; RYAN; MEREDITH; LIPSITZ; EVANS, 1990; FRONTERA; MEREDITH; O'REILLY; KNUTTGEN; EVANS, 1998; HAKKINEN; KALLINEN; IZQUIERDO; JOKELAINEN; LASSILA; MALKIA; KRAEMER; NEWTON; ALEN, 1998; HAKKINEN; ALEN; KALLINEN; NEWTON; KRAEMER, 2000).

Entretanto, não há na literatura estudos sobre os possíveis mecanismos que ocasionam 0 aumento da força muscular com aplicações sucessivas de testes de 1RM. No entanto, uma vez que $\mathrm{o}$ procedimento de familiarização em nosso estudo foi realizado em três e quatro sessões (para as mulheres e os homens, respectivamente), além do aprendizado do movimento, é razoável considerar que os mecanismos envolvidos sejam semelhantes aos verificados nas primeiras semanas de um programa de TF, como a melhora das coordenações intra e intermuscular, já comprovadas por alguns estudos (CAROLAN; CAFARELLI, 1992; PUCCI; GRIFFIN; CAFARELLI, 2006; SALE, 1988). De acordo com isso, se o teste de 1RM for realizado em estudos que têm como objetivo analisar os ganhos de força decorrentes das adaptações neurais, realizar a familiarização ao teste de $1 \mathrm{RM}$ até a estabilização da carga poderá subestimar os efeitos do TF usado como intervenção. Por outro lado, a familiarização até a estabilização seria apropriada para as investigações interessadas em avaliar os ganhos de força decorrentes apenas da hipertrofia muscular.

\section{Conclusões}

De acordo com nossos resultados, indivíduos inexperientes em TF podem atingir a estabilização da carga do teste de $1 \mathrm{RM}$ no exercício agachamento realizando entre três e quatro sessões de avaliação. Isto pode ser particularmente verdadeiro para o agachamento realizado na barra guiada.

\section{Referências}

BENTON, M. J.; SWAN, P. D.; PETERSON, M. D. Evaluation of multiple one repetition maximum strength trials in untrained women. Journal of Strength and Conditioning Research, Arizona, v. 23, n. 5, p. 1501-1507, 2009.

BORG, G. Borg's perceived exertion and pain scales. Stockholm: Human Kinetics, 1998.

BROWN, L. E.; WEIR, J. P. Procedures recommendation I: accurate assessment of muscular strength and power. Journal of Exercise Physiology, Arkansas, v. 4, n. 3, p.121, 2001.

CAROLAN, B.; CAFARELLI, E. Adaptations in coactivation after isometric resistance training. Journal of Applied Physiology, Bethesda, v. 73, n. 3, p. 911-917, 1992.

CRONIN, J. B.; HENDERSON, M. E. Maximal strength and power assessment in novice weight trainers. Journal of Strength and Conditioning Research, Auckland, v. 18, n. 1, p. 48-52, 2004.

DIAS, R. M. R.; CYRINO, E. S.; SALVADOR, E. P; CALDEIRA, L. F. S; NAKAMURA, F. Y.; PAPST, R. R.; BRUNA, N.; GURJÃO, A. L. D. Influência do processo de familiarização para avaliação da força muscular em testes de 1-RM. Revista Brasileira de Medicina do Esporte, Londrina, v. 11, n. 1, p. 34-38, 2005.

FIATARONE, M. A.; MARKS, E. C.; RYAN, N. D.; MEREDITH, C. N.; LIPSITZ, L. A.; EVANS, W. J. High-intensity strength training in nonagenarians. Effects on skeletal muscle. Journal of the American Medical Association, Massachusetts, v. 263, n. 22, p.3029-3034, 1990.

FRONTERA, W. R.; MEREDITH, C. N.; O'REILLY, K. P.; KNUTTGEN, H. G.; EVANS, W. $J$. Strength conditioning in older men: skeletal muscle hypertrophy and improved function. Journal of Applied Physiology, Massachusetts, v. 64, n. 22, p. 1038-1044, 1998. 
GLASS, S. C.; STANTON, D. R. Self-selected resistance training intensity in novice weightlifters. Journal of Strength and Conditioning Research, Wayne, v. 18, n. 2, p. 324-327, 2004.

GURJÃO, A. L. D.; CYRINO, E. S.; CALDEIRA, L. F. S.; NAKAMURA, F. Y.; OLIVEIRA, A. R.; DIAS, R. M. R.; SALVADOR, E. P. Variação da força muscular em testes repetitivos de 1-RM em crianças pré-puberes. Revista Brasileira de Medicina do Esporte, Londrina, v. 11, n. 6, p. 319-324, 2005.

HAKKINEN, K.; ALEN, M.; KALLINEN, M.; NEWTON, R. U.; KRAEMER, W. J.

Neuromuscular adaptation during prolonged strength training, detraining and restregthentraining in middle-aged and elderly people.

European Journal of Applied Physiology, Jyvaskyla, v. 83, n. 1, p. 51-62, 2000

HAKKINEN, K.; KALLINEN, M.; IZQUIERDO, M.; JOKELAINEN, K.; LASSILA, H.; MALKIA, E.; KRAEMER, W. J.; NEWTON, R. V.; ALEN, M. Changes in agonist-antagonista EMG, muscle CSA, and force during strength training in middleaged and older people. Journal of Applied Physiology, Bethesda, v. 84, n. 4, p. 1341-1349, 1998.

LEVINGER, I.; GOODMAN, C.; HARE, D. L.; JERUMS, G.; TOIA, D.; SELIG, S. The reliability of the 1-RM strength test for untrained middleaged individuals. Journal of Science and Medicine in Sport, Austin, v. 12, n. 2, p. 310-316, 2009.

NIEWIADOMSKI, W.; LASKOWSKA, D.; GASIOROWS, K. A.; CYBULSKI, G.; STRASZ, A.; LANGFORT, J. Determination and prediction of one repetition maximum: safety considerations. Journal of Human Kinetics, Varsóvia, v. 19, p.109-120, Mar. 2008.

PHILLIPS, W. T.; BATTERHAM, A. M.; VALENZUELA, J. E.; BURKETT, L. N.; RELIABILITY, O. F. Maximal strength testing in older adults. American Academy of Physical Medicine and Rehabilitation, Arizona, v. 85, n. 2, p. 329-334, 2004.

PLOUTZ-SNYDER, L. L.; GIAMIS, E. L. Orientation and familiarization to $1 \mathrm{RM}$ strength testing in old and young women. Journal of Strength and Conditioning Research, New York, v. 15, n. 4, p. 519-23, 2001.

PUCCI, A. R.; GRIFFIN, L.; CAFARELLI, E. Maximal motor unit firing rates during isometric resistance training in men. Experimental Physiology, Toronto, v. 91, n. 1, p. 171-178, 2006.
RATAMESS, N. A.; ALVAR, B. A.; EVETOCH, T. K.; HOUSH, T. J.; KIBLER, W. B.; KRAEMER, W. J.; TRIPLETT, M. T. Progression models in resistance training for healthy adults. American College of Sports Medicine position stand. Medicine and Science in Sports and Exercise, Indianapolis, v. 41, n. 3, p. 687-708, 2009.

REINKING, M. F.; BOCKRATH-PUGLIESE, K.; WORRELL, T.; KEGERREIS, R. L.; MILLERSAYERS, K.; FARR, J. Assessment of quadriceps muscle performance by hand-held, isometric, and isokinetic dynamometry in patients with knee dysfunction. Journal of Orthopedic \& Sports Physical Therapy, Dubuque, v. 24, n. 3, p. 154159, 1996.

RYDWIK, E.; KARLSSON, C.; FRANDIN, K.; AKNER, G. Muscle strength testing with one repetition maximum in the arm/shoulder for people aged $75+-$ test-retest reliability. Clinical

Rehabilitation, Jarfalla, v. 21, n. 3, p. 258-265, 2007.

SALE, D. G. Neural adaptation to resistance training. Medicine and Science in Sports and Exercise, Indianapolis, v. 20, n. 5, p. 135-145, 1988.

SOARES-CALDEIRA, L. F.; DIAS, R. M. R.; OKUNO, N. M.; CYRINO, E. S.; GURJÃO, A. L. D.; PLOUTZ-SNYDER, L. L. Familiarization indexes in sessions of 1-RM tests in adult women. Journal of Strength and Conditioning Research, Londrina, v. 23, n. 7, p. 2039-2045, 2009.

Endereço:

Carla Silva-Batista.

Av. Prof. Mello Moraes, 65 Butantã

Cidade. Universitária,

São Paulo SP Brasil

05508-030

Telefone: (11) 3091.8796

Fax: (11) 3091.8733

email: carla_sbatista@hotmail.com

Recebido em: 6 de agosto de 2010. Aceito em: 20 de abril de 2011.

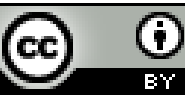

Motriz. Revista de Educação Física. UNESP, Rio Claro, SP, Brasil - elSSN: 1980-6574 - está licenciada sob Creative Commons - Atribuição 3.0 